\title{
p53 thwarts the editors
}

Most methods for editing the genome start by making a targeted double-strand break, which is then stitched up by one of several cellular DNA repair pathways. But cells possess other mechanisms to react to DNA damage. In mammalian cells, a central player is p53, which defends against genomic damage by inducing cell cycle arrest or apoptosis. In two recent papers in Nature Medicine, Ihry et al. ${ }^{1}$ and Haapaniemi et al. ${ }^{2}$ show that p53 is activated after DNA cleavage by the RNAguided CRISPR-Cas9 nuclease and that it impairs cell growth and survival, at least in some cell types.

Ihry et al. ${ }^{1}$ investigated the utility of the Cas9 system in editing the genome of human pluripotent stem cells (hPSCs). Although editing efficiencies were very high, the authors also noticed a sharp decrease in the number of viable cells and in cell growth in samples treated with various guide RNAs, leading to a $60-95 \%$ reduction in cell confluency after 7 days. The effect was observed after viral transduction of two different inducible constructs and after transfection with Cas9 ribonucleoproteins. The use of a high-fidelity Cas9 variant did not help, suggesting that off-target cleavage was not a major contributor. When the authors screened a library of 13,000 guide RNAs, cells expressing Cas9 showed substantially reduced growth compared with cells lacking Cas9. The final pool of guide RNAs was enriched for sequences that had no genomic target, suggesting that merely inducing double-strand breaks causes toxicity in hPSCs.

Not surprisingly, p53 pathway genes were substantially upregulated in the edited cells, and p53 was computationally identified as the most prominent key regulator. Indeed, targeting p53 with specific guide RNAs reduced toxicity to cells. Similarly, the low efficiency of precise genome editing using Cas9 and homology-directed repair (HDR) was sharply increased by inhibition of p53 with a dominant negative $\mathrm{p} 53$ mutant.

Similar effects were observed by Haapaniemi et al. ${ }^{2}$ in one somatic cell type.
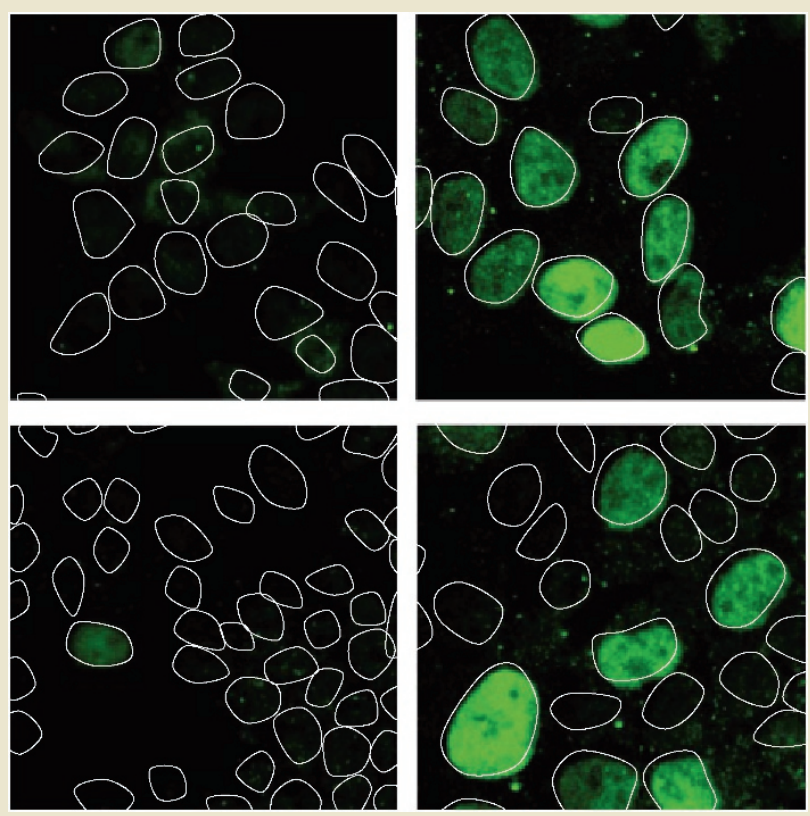

Reprinted by permission from Ihry et al., reference 1 .

Performing a negative-selection screen in immortalized human retinal pigment epithelium (RPE1) cells, they observed a substantial increase in guide RNAs targeting p53 and several of its downstream effectors. Guide RNAs targeting essential genes were not efficiently depleted, however, again suggesting a nonspecific p53-mediated toxicity caused by the double-strand break itself and not by the specific targets of guide RNAs. The key role of $\mathrm{p} 53$ is corroborated by the fact that, in $\mathrm{p} 53^{-/-} \mathrm{RPE} 1$ cells, essential genes were recovered as the most prominent hits. As in the paper by Ihry et al. ${ }^{1}$, p53 activation proved limiting to precision genome editing with HDR, and inhibition of p53 activity using its antagonist MDM2 substantially increased the frequency of successful HDR repair events.

Currently, the broader implications of the findings are unclear as only a few cell lines were studied ${ }^{1,2}$. "Transient p53 inhibition might prove useful for screening applications in some cell lines," says Keith Joung of the Massachusetts
General Hospital, "and it might be possible to use it to increase the efficiency of ex vivo genome editing in clinical applications, if it proves to be a problem for a given cell type and if the safety of transient p53 inhibition can be thoroughly established." Whether the findings raise safety concerns for clinical applications also remains unclear. Although an enrichment of preexisting p53-defective cells is possible, especially if successfully edited cells are selected for in vivo or in vitro, and might facilitate tumorigenesis, this was not shown in the papers. As Fyodor Urnov of the Altius Institute notes, "One should not forget that genome editing is in the clinic and safety has been thoroughly tested in the relevant cell types and organs before the clinical trials were initiated."

\section{Markus Elsner,} Senior Editor

1. Ihry, R.J. et al. Nat. Med. 24, 939-946 (2018).

2. Haapaniemi, E., Botla, S., Persson, J., Schmierer, B. \& Taipale, J. Nat. Med. 24, 927-930 (2018). 